\title{
The impact of chemotherapy on cognitive outcomes in adults with primary brain tumors
}

\author{
Abrey, L E
}

\begin{abstract}
There is growing recognition that chemotherapy may have short and long term impact on cognitive function of cancer patients. However, the impact of chemotherapy on the cognition of adult patients with primary brain tumor has not been extensively studied. This article will review the evidence for both positive and negative impact of chemotherapy on cognitive function of adult brain tumor patients as well as potential confounding factors.
\end{abstract}

DOI: https://doi.org/10.1007/s11060-012-0807-6

Posted at the Zurich Open Repository and Archive, University of Zurich

ZORA URL: https://doi.org/10.5167/uzh-67364

Journal Article

Published Version

Originally published at:

Abrey, L E (2012). The impact of chemotherapy on cognitive outcomes in adults with primary brain tumors. Journal of Neuro-Oncology, 108(2):285-290.

DOI: https://doi.org/10.1007/s11060-012-0807-6 


\title{
The impact of chemotherapy on cognitive outcomes in adults with primary brain tumors
}

\author{
Lauren E. Abrey
}

Received: 20 July 2011/ Accepted: 26 January 2012/Published online: 17 February 2012

(C) Springer Science+Business Media, LLC. 2012

\begin{abstract}
There is growing recognition that chemotherapy may have short and long term impact on cognitive function of cancer patients. However, the impact of chemotherapy on the cognition of adult patients with primary brain tumor has not been extensively studied. This article will review the evidence for both positive and negative impact of chemotherapy on cognitive function of adult brain tumor patients as well as potential confounding factors.
\end{abstract}

Keywords Cognition - Chemo-brain · Chemotherapy · Primary brain tumor · Glioblastoma · Primary CNS lymphoma $\cdot$ Oligodendroglioma

\section{Introduction}

The impact of chemotherapy on cognitive outcomes of cancer survivors is increasingly recognized and frequently referred to as "chemo brain" [1]. While this concept has gained popularity it is still being defined and verified. First described in the 1980s most early reports were attributed to patients surviving aggressive therapy for breast cancer and lymphoma. Initial descriptions were largely based on selfreporting by patients who described difficulties with memory and concentration. Increasingly studies and reports have become more rigorous with prospective neuro-cognitive assessments that document pre and post treatment deficits across multiple domains [2]. Although attention, concentration and memory remain primary areas of impairment other cognitive dimensions are also recognized as having

L. E. Abrey ( $₫)$

Department of Neurology, University Hospital Zurich,

Frauenklinikstrasse 26, 8091 Zurich, Switzerland

e-mail: lauren.abrey@usz.ch been affected by chemotherapy such as visuo-spatial and primary visual pathways [3].

The mechanism of injury by which chemotherapy results in cognitive loss is not known and is likely to vary according to the specific agent used. In animal models increased cell death or decreased cell division in the subventricular zone and dentate gyrus have been described following exposure to carmustine, cisplatin, cytarabine and methotrexate $[4,5]$. These models suggest vulnerability of neural stem cell populations with resultant cognitive impact. Primary pathologic lesions including demyelination, inflammation and microvascular injury have all been postulated as mechanisms underlying neurotoxicity of therapy [6]. Synergistic injury may occur with concomitant or sequential administration of brain radiotherapy and chemotherapy because of alterations in blood brain barrier permeability and drug distributions. Finally a recent report suggests that brain volume may decrease following myeloablative chemotherapy prior to stem cell transplant in leukemia and multiple sclerosis patients [7].

While "chemo brain" is increasingly recognized in patients with cancer other than breast cancer and lymphoma the question for this manuscript is whether or not adult brain tumor patients are at risk for significant cognitive sequelae of chemotherapeutic treatment. This topic has not been extensively explored in the past in large part due to the poor prognosis of brain tumor patients and the relative lack of effective chemotherapeutic agents.

\section{Confounding factors}

Before considering whether or not brain tumor patients suffer adverse consequences of chemotherapy administration it is important to consider the other factors that impact cognitive 
function in this patient population. Tumor location is perhaps the most obvious consideration. The majority of primary brain tumors are located in the cerebral hemispheres and have an impact on some aspect of intellectual function. Although this may be subtle or unrecognized by the patient, family and treating physician, particularly during the acute phase of diagnosis and early treatment, it may be increasingly apparent in the long-term survivor who attempts to return to work or full level of activity following successful treatment [8].

Age is a critical consideration in assessment of cognition. The average age of diagnosis for glioblastoma, the most common primary brain tumor of adults, is 60 years. Normal persons in this age range may experience some normal slowing of information processing and retrieval as well as complaints of short-term memory loss. Furthermore in older patients, co-morbidities and associated medications may have a direct or indirect impact on cognition that must be considered. Finally as MR imaging is often used as a surrogate measure of neurotoxicity it must be recognized that nonspecific white matter changes are seen with increasing frequency according to patient age. In fact, age was one of the strongest determinants of cognitive outcome in a series of 79 primary brain tumor patients assessed at UCLA [9].

The presence or absence of both seizures and anti-epileptics medications is another important consideration in the assessment of cognitive outcome. Approximately one in three brain tumor patients will have a seizure during the course of their illness and it is known that seizure activity has a direct impact on cognitive function. Furthermore the use of anti-epileptic drugs is known to cause psychomotor slowing, decreased working memory and general cognitive decline in patients with a primary brain tumor [10].

Other considerations include the presence of co-morbidities with a direct impact on the CNS such as depression and dementia. Supportive medications may also have a significant impact on cognition. Corticosteroids are likely the biggest culprit as they are the most frequently used and have a wide range of CNS impacts from mood changes (mania and depression), insomnia, psychosis and impaired memory $[11,12]$.

Finally the other definitive therapies used for management of the brain tumor may result in long-term cognitive sequelae. Surgical resection can alleviate acute neurologic symptoms but may also result in permanent deficit from local injury. Radiotherapy often has a delayed impact on white matter and neurons that may result in acute or delayed leukoencephalopathy with or without associated cognitive dysfunction [10, 13].

\section{Specific impact of chemotherapy}

A number of different scenarios may be considered to assess the impact of chemotherapy on cognitive outcome of primary brain tumor patients. Although the use of chemotherapy alone is an uncommon strategy for primary brain tumors some patients with chemosensitive PCNSL or oligodendroglioma have been successfully managed in this fashion and provide some useful observations. Combinations of chemotherapy and radiotherapy allow for insights about sequence, specific agents and high-risk populations. Finally the increasing use of neuro-cognitive testing as a prospective endpoint in some clinical trials will allow more confident analysis of the data and development of better treatment strategies in the future.

\section{Chemotherapy alone}

\section{Primary CNS lymphoma (PCNSL)}

Altered mentation is one of the most frequent ways for a patient with PCNSL to present. Indeed detailed neurocognitive testing at baseline and prior to specific anti-tumor treatment in PCNSL demonstrates that most patients have impairment in one or more of the following domains: attention, executive function, memory, psychomotor speed or language (specifically naming or fluency) [14]. A number of small studies have now been done that specifically incorporate prospective neuro-cognitive testing as part of PCNSL clinical trials [15-21]. These studies have consistently shown that patients either remain stable or have clear cognitive improvement during the course of initial chemotherapy provided that their tumor is responding to treatment. This includes trials with alternate and intensive delivery strategies for chemotherapy such as blood brain barrier disruption and dose intensive chemotherapy used for myeloablation [16, 22]. In long term follow up of cognitive function it has been shown that PCNSL patients treated with chemotherapy alone most often remain cognitively stable although some studies have reported minor or occasional declines in attention, memory and verbal fluency [14].

One retrospective study compared cognitive outcome in PCNSL patients treated at the same institution with either chemotherapy alone or in combination with RT [23]. Twenty-four patients were treated with a combination of chemotherapy and RT while 26 patients received chemotherapy alone. When controlling for age and time from initial diagnosis the authors concluded that patients treated with chemotherapy alone had substantially less impairment than those treated with both chemotherapy and radiotherapy. Furthermore the degree of impairment in those patients treated with chemotherapy alone was more likely to be within one standard deviation of the norm suggesting a less significant impact on day-to-day functioning. 
Anaplastic oligodendroglioma

There is growing evidence to suggest that patients with anaplastic oligodendroglioma with deletion of $1 \mathrm{p}$ and/or $19 q$ may be successfully treated with chemotherapy alone as initial therapy $[24,25]$. One motivation for this treatment strategy comes from the fact that these tend to be young patients often with large tumors and a relatively long life expectancy. Therefore, the concept is that delaying or deferring radiotherapy might minimize cognitive impact of therapy. Despite this trend there has been little in the way of prospective cognitive assessment in this patient population.

One study that treated such patients with chemotherapy alone using a myeloablative chemotherapy regimen in place of usual consolidation radiotherapy described that 21 long-term survivors had largely returned to their pre-morbid career or level of function [26]. Two patients were noted to have impairments ascribed to tumor but possibly treatment related. A large ongoing intergroup trial in this patient population will test chemotherapy alone as an initial treatment strategy and prospective cognitive testing is incorporated as a composite primary endpoint [27].

\section{Low grade glioma}

A recent recommendation from the EFNS-EANO task force suggests that chemotherapy alone may be used as initial therapy for patients with large residual or unresectable tumor to delay the risk of late neurotoxicity associated with large field radiotherapy, particularly when $1 \mathrm{p}$ and $19 \mathrm{q}$ loss is present, based on level B evidence [28]. Similar to patients with anaplastic oligodendroglioma, patients with low grade glioma are often in the prime of their life and may live for many years with their tumor; hence preservation of cognitive function is a critical goal of therapy. Several studies have suggested that chemotherapy may result in various clinical improvements including improved seizure control, neurologic function and quality of life [29-31]; however, detailed neurocognitive evaluation of low grade glioma patients treated with chemotherapy alone is sparse. Correa et al. included three patients treated with chemotherapy alone in a longitudinal analysis of cognitive function in low grade glioma but this subset was too small to be critically compared [32]. Increasingly there is a mandate for clinical trials in this patient population to assess cognitive function as a prospective endpoint [28, 33]. The ongoing EORTCNCIC study for low grade glioma includes a chemotherapy alone arm and incorporates MMSE and QOL testing with one objective to assess the delayed toxicity of temozolomide alone [34]. Another ongoing cooperative group study sponsored by ECOG will assess the addition of temozolomide to radiotherapy for newly diagnosed low grade glioma; a detailed neurocognitive substudy is planned [35].

\section{Chemotherapy and radiotherapy}

Most often patients with primary brain tumors are treated with some combination of chemotherapy and radiotherapy. These treatments may be given in sequence or concomitantly. Depending on the underlying pathology, radiotherapy dose and field, and particular chemotherapeutic agent used, a variety of different outcomes may be anticipated.

\section{Primary CNS lymphoma}

PCNSL was one of the first primary brain tumors to herald the risk of cognitive impact of therapy. Recognition of the detrimental impact of combined chemoradiotherapy following the sequential administration of high dose MTX and whole brain radiotherapy was first described in the early 1990s. Although this combination was clearly superior to the use of radiotherapy alone in terms of disease control and potential to extend survival, with prolonged survival it became clear that survivors, particularly those over the age of 60 , were at high risk of treatment related dementia [10, 14].

A detailed review of multiple studies in the literature shows diffuse impairments in cognition following standard combined therapy with high dose methotrexate and radiotherapy [14]. The pattern of cognitive deficits spans multiple domains including attention and executive functions, psychomotor speed, learning and retrieval of new information. While the direct attribution of cognitive toxicity to the individual components of therapy is not possible from this data it seems likely that the particular sequence and combination of methotrexate followed by whole brain radiotherapy is largely responsible as opposed to either component alone.

\section{Anaplastic oligodendroglioma}

Similar to PCNSL, patients with anaplastic oligodendroglioma have the potential for prolonged survival with excellent disease control and this tumor particularly in the setting of $1 p$ and $19 q$ deletion is known to be sensitive to chemotherapy. Further, patients with oligodendroglioma are often younger with a median age of 30-40 and therefore are in the prime of their working and productive life making cognitive function of critical importance.

Several studies have looked at combinations of chemotherapy and radiation to optimize disease control and survival. In particular two recently published studies compared radiation alone to radiation in combination with PCV chemotherapy [36, 37]. Both studies found similar survival for each treatment arm and confirmed the prognostic impact of $1 \mathrm{p}$ and $19 \mathrm{q}$ deletion on overall survival. While neither study included cognitive function as a 
prospective endpoint for patient outcomes each study examined potential surrogate endpoints, such as mini mental status examination, performance status and quality of life. The RTOG study found that MMSE and brain related quality of life were similar between the two treatment arms [38]. However in both groups these scores tended to decline overtime suggesting the potential for chronic deleterious impact of therapy but not specific to chemotherapy. It was also observed that better scores were associated with improved survival. Similarly in the EORTC study no significant difference in QOL was observed between the two treatment groups outside of the acute treatment interval suggesting no chronic negative impact from the addition of chemotherapy [39].

\section{Glioblastoma}

Previously the survival of patients with newly diagnosed GBM was too short to meaningfully assess the chronic or cognitive impact of relatively ineffective or short-lived therapies. However, increasingly therapies are available which result in meaningful survival as evidenced by the number of long-term survivors.

Assessment of long-term survivors reveals some important observations that may be used to guide future therapeutic strategies [40, 41]. One study of 39 GBM patients with survival of $3 y$ or more following initial diagnosis showed that the majority of patients $(85 \%)$ had neurologic impairment, including at least $30 \%$ with cognitive dysfunction [41]. Nine patients had evidence of a cerebrovascular event, either ischemic or hemorrhagic, and six had radiation-induced necrosis. It is important to consider that most of these patients $(72 \%)$ had been treated both for their primary diagnosis of GBM as well as for subsequent recurrence with a variety of therapies.

The addition of temozolomide during initial radiotherapy has been the major change in standard of care over the past decade [42]. Patients receiving this treatment have more than double the possibility of surviving at 2 years as compared with patients treated with radiation alone. This treatment approach raises the question of the safety of administration of concomitant chemoradiotherapy. From the long term follow up of the pivotal study of the EORTC there is little evidence to suggest additive or synergistic toxicity resulting from this regimen [43]. Indeed, only three patients out of the original cohort are reported as having late treatment related toxicity at 5 years. Two patients in the concurrent temozolomide and radiotherapy arm had late toxicity-one patient with seizures and another with visual deficit; one patient in the RT alone arm is reported to have fatigue. These results are further supported by a small prospective study from the Netherlands that directly assessed cognitive function in newly diagnosed patients being treated with this standard therapy [44]. Early assessment during and immediately after the therapy found that most patients were cognitively stable or improved; however, long-term follow up was not available.

This new standard of care is currently under debate for older patients. Patients over the age of 70 were not included in the original EORTC study. This is an important area of consideration because older patients comprise a significant fraction of patients diagnosed with GBM. Furthermore this patient subset typically has a worse prognosis and may be more vulnerable to the negative effects of therapy. Several studies and reports have now demonstrated that concomitant TMZ/RT may be an effective therapy in older patients [45-48]. However a recent prospective study suggests that there may be a significant risk of toxicity among older patients treated with this regimen [46]. Fifty-eight patients over the age of 65 were treated with the standard of care and achieved excellent progression free and overall survival (9.5 and 13.7 months, respectively) as compared with historic controls; however, $25 \%$ of patients developed grade 3-4 cognitive dysfunction and $10 \%$ developed leukoencephalopathy. The authors concluded that this rate of toxicity may be unacceptably high and merits further study prior to recommending this regimen as standard of care in older patients. Several other reports have raised similar concerns of excess toxicity among older patients treated with concurrent temozolomide and RT [47, 48]; an ongoing intergroup study will provide prospective data with regard to efficacy and toxicity in this population [49]. Of interest, a recent prospective study of temozolomide alone for frail patients over 70 years of age showed improvement of QOL and cognitive function in responding or stable patients [50].

A recent study looked at neurocognitive outcome as an exploratory endpoint for a trial of bevacizumab in recurrent glioblastoma [51]. Using three standardized tests of specific cognitive domains the researchers were able to demonstrate that neurocognitive status (worsened, stable, or improved) correlated with radiographic tumor response and disease control as measured by progression free survival. This data suggests the possibility of incorporating neurocognitive testing as an alternate measure of efficacy to support imaging criteria. Neuro-cognitive function is being used as an endpoint in several ongoing clinical trials of new therapies for brain tumors.

\section{Conclusions}

At the present time there is little evidence to support a serious concern for the risk of "chemo brain" among patients with a primary brain tumor. While the acute impact of chemotherapy may result in fatigue and apparent 
cognitive decline during the course of active therapy, available data suggests that most patients with a positive response to chemotherapy will also have cognitive stability or improvement to mirror the anti-tumor effect. Data regarding the long-term cognitive impact of chemotherapy remains sparse. The best data from patients with PCNSL do not support significant delayed or chronic neurotoxicity induced by chemotherapy. Long term follow up of patients with anaplastic oligodendroglioma that fail to suggest any incremental decline in function among patients treated with chemotherapy in addition to RT; but detailed cognitive assessment following chemotherapy is not available for these patients or those with low grade glioma. Emerging data related to elderly patients with glioblastoma merit additional study to exclude significant neurotoxicity as a result of concomitant temozolomide and radiotherapy. Our primary charge continues to be the discovery of more effective therapies to control primary brain tumors as the major risk to cognitive function at present is the presence of active tumor either at diagnosis and recurrence.

Conflict of interest statement The author declares that she has no conflict of interest.

\section{References}

1. Janelsins MC, Kohli S, Mohile SG et al (2011) An update on cancer- and chemotherapy-related cognitive dysfunction: current status. Semin Oncol 38:431-438

2. Wefel JS, Vardy J, Ahles T, Schagen SB (2011) International cognition and cancer task force recommendations to harmonise studies of cognitive function in patients with cancer. Lancet Oncol 12:703-708

3. Raffa RB, Tallarida RJ (2010) Effects on the visual system might contribute to some of the cognitive deficits of cancer chemotherapy-induced 'chemo-fog'. J Clin Pharm Ther 35:249-255

4. Dietrich J, Han R, Yang Y et al (2006) CNS progenitor cells and oligodendrocytes are targets of chemotherapeutic agents in vitro and vivo. J Biol 5:1-23

5. Dietrich J, Monje M, Wefel J et al (2008) Clinical patterns and biological correlates of cognitive dysfunction associated with cancer therapy. Oncologist 13:1285-1295

6. Saykin AJ, Ahles TA, McDonald BC (2003) Mechanisms of chemotherapy-induced cognitive disorders: neuropsychological, pathophysiological, and neuroimaging perspectives. Semin Clin Neuropsychiatry 8:201-216

7. Petzold A, Mondria T, Kuhle J et al (2010) Evidence for acute neurotoxicity after chemotherapy. Ann Neurol 68:806-815

8. Lucas MR (2010) The impact of chemo brain on the patient with a high-grade glioma. Adv Exp Med Biol 678:21-25

9. Kaleita TA, Wellisch DK, Cloughesy TF et al (2004) Prediction of neurocognitive outcome in adult brain tumor patients. J Neurooncol 67:245-253

10. Correa DD (2010) Neurocognitive function in brain tumors. Curr Neurol Neurosci Rep 10:232-239

11. Fietta P, Fietta P, Delsante G (2009) Central nervous system effects of natural and synthetic glucocorticoids. Psychiatry Clin Neurosci 63(5):613-622
12. Baile WF (1996) Neuropsychiatric disorders in cancer patients. Curr Opin Oncol 8(3):182-187

13. Béhin A, Delattre JY (2004) Complications of radiation therapy on the brain and spinal cord. Semin Neurol 24(4):405-417

14. Correa DD, Maron L, Harder $\mathrm{H}$ et al (2007) Cognitive functions in primary central nervous system lymphoma: literature review and assessment guidelines. Ann Oncol 18:1145-1151

15. Sandor V, Stark-Vancs V, Pearson D et al (1998) Phase II trial of chemotherapy alone for primary CNS and intraocular lymphoma. J Clin Oncol 16:3000-3006

16. Correa DD, Anderson ND, Glass A et al (2003) Cognitive functions in primary central nervous system lymphoma patients treated with chemotherapy and stem cell transplantation: preliminary findings. Clin Adv Hematol Oncol 1:490

17. Fliessbach K, Urbach H, Helmstaedter C et al (2003) Cognitive performance and magnetic resonance imaging findings after highdose systemic and intraventricular chemotherapy for primary central nervous system lymphoma. Arch Neurol 60:563-568

18. Fliessbach K, Helmstaedter C, Urbach H et al (2005) Neuropsychological outcome after chemotherapy for primary CNS lymphoma: a prospective study. Neurology 64:1184-1188

19. Pels H, Schmidt-Wolf IGH, Glasmacher A et al (2003) Primary central nervous system lymphoma: results of a pilot study and phase II study of systemic and intraventricular chemotherapy with deferred radiotherapy. J Clin Oncol 21:4489-4495

20. Schlegel U, Pels H, Glasmacher A et al (2001) Combined systemic and intraventricular chemotherapy in primary CNS lymphoma: a pilot study. J Neurol Neurosurg Psychiatr 71:118-122

21. Herrlinger U, Kuker W, Uhl M et al (2005) NOA-03 trial of highdose methotrexate in primary central nervous system lymphoma: final report. Ann Neurol 57:843-847

22. McAllister LD, Doolittle ND, Guastadisegni PE et al (2000) Cognitive outcomes and long-term follow-up results after enhanced chemotherapy delivery for primary central nervous system lymphoma. Neurosurgery 46:51-60

23. Correa DD, DeAngelis LM, Shi W et al (2004) Cognitive functions in survivors of primary central nervous system lymphoma. Neurology 62:548-555

24. Wick W, Hartmann C, Engel C et al (2009) NOA-04 randomized phase III trial of sequential radiochemotherapy of anaplastic glioma with procarbazine, lomustine, and vincristine or temozolomide. J Clin Oncol 27:5874-5880

25. Lassman AB, Iwamoto FM, Cloughesy TF et al (2011) International retrospective study of over 1000 adults with anaplastic oligodendroglial tumors. Neuro Oncol 13:649-659

26. Abrey LE, Childs BH, Paleologos N et al (2006) High-dose chemotherapy with stem cell rescue as initial therapy for anaplastic oligodendroglioma: long-term follow-up. Neuro Oncol 8:183-188

27. A phase III intergroup study of radiotherapy versus temozolomide alone versus radiotherapy with concomitant and adjuvant temozolomide for patients with $1 \mathrm{p} / 19 \mathrm{q}$ codeleted anaplastic glioma. ClinicalTrials.gov identifier: NCT00887146

28. Soffietti R, Baumert BG, Bello L (2010) European federation of neurological societies. Guidelines on management of low-grade gliomas: report of an EFNS-EANO task force. Eur J Neurol 17(9):1124-1133

29. Mason WP, Krol GS, DeAngelis LM (1996) Low-grade oligodendroglioma responds to chemotherapy. Neurology 46(1):203207

30. Brada M, Viviers L, Abson C et al (2003) Phase II study of primary temozolomide chemotherapy in patients with WHO grade II gliomas. Ann Oncol 14(12):1715-1721

31. Kesari S, Schiff D, Drappatz J et al (2009) Phase II study of protracted daily temozolomide for low-grade gliomas in adults. Clin Cancer Res 15(1):330-337 
32. Correa DD, Shi W, Thaler HT et al (2008) Longitudinal cognitive follow-up in low grade gliomas. J Neurooncol 86(3):321-327

33. van den Bent MJ, Wefel JS, Schiff D et al (2011) Response assessment in neuro-oncology (a report of the RANO group): assessment of outcome in trials of diffuse low-grade gliomas. Lancet Oncol 12(6):583-593

34. A phase III randomized study of radiotherapy versus temozolomide in patients with low-grade gliomas. ClinicalTrials.gov identifier: NCT00182819

35. A phase III study of radiation therapy with or without temozolomide for symptomatic or progressive low-grade gliomas. ClinicalTrials.gov identifier: NCT00978458

36. van den Bent MJ, Carpentier AF, Brandes AA et al (2006) Adjuvant procarbazine, lomustine, and vincristine improves progression-free survival but not overall survival in newly diagnosed anaplastic oligodendrogliomas and oligoastrocytomas: a randomized European Organisation for Research and Treatment of Cancer phase III trial. J Clin Oncol 24:2715-2722

37. Cairncross G, Berkey B, Shaw E et al (2006) Phase III trial of chemotherapy plus radiotherapy compared with radiotherapy alone for pure and mixed anaplastic oligodendroglioma: intergroup radiation therapy oncology group trial 9402. J Clin Oncol 24:2707-2714

38. Wang M, Cairncross G, Shaw E et al (2010) Cognition and quality of life after chemotherapy plus radiotherapy (RT) vs. RT for pure and mixed anaplastic oligodendrogliomas: radiation therapy oncology group trial 9402. Int J Radiat Oncol Biol Phys 77:662-669

39. Taphoorn MJ, van den Bent MJ, Mauer ME et al (2007) Healthrelated quality of life in patients treated for anaplastic oligodendroglioma with adjuvant chemotherapy: results of a European Organisation for research and treatment of cancer randomized clinical trial. J Clin Oncol 25:5723-5730

40. Steinbach JP, Blaicher HP, Herrlinger U et al (2006) Surviving glioblastoma for more than 5 years: the patient's perspective. Neurology 66:239-242

41. Hottinger AF, Yoon H, DeAngelis LM, Abrey LE (2009) Neurological outcome of long-term glioblastoma survivors. J Neurooncol 95:301-305
42. Stupp R, Mason WP, van den Bent MJ et al (2005) Radiotherapy plus concomitant and adjuvant temozolomide for glioblastoma. N Engl J Med 352:987-996

43. Stupp R, Hegi ME, Mason WP et al (2009) Effects of radiotherapy with concomitant and adjuvant temozolomide versus radiotherapy alone on survival in glioblastoma in a randomised phase III study: 5-year analysis of the EORTC-NCIC trial. Lancet Oncol 10:459-466

44. Hilverda K, Bosma I, Heimans JJ et al (2010) Cognitive functioning in glioblastoma patients during radiotherapy and temozolomide treatment: initial findings. J Neurooncol 97:89-94

45. Scott JG, Suh JH, Elson P et al (2011) Aggressive treatment is appropriate for glioblastoma multiforme patients 70 years old or older: a retrospective review of 206 cases. Neuro Oncol 13:428-436

46. Brandes AA, Franceschi E, Tosoni A et al (2009) Temozolomide concomitant and adjuvant to radiotherapy in elderly patients with glioblastoma: correlation with MGMT promoter methylation status. Cancer 115:3512-3518

47. Minniti G, De Sanctis V, Muni R et al (2008) Radiotherapy plus concomitant and adjuvant temozolomide for glioblastoma in elderly patients. J Neurooncol 88:97-103

48. Sijben AE, McIntyre JB, Roldán GB et al (2008) Toxicity from chemoradiotherapy in older patients with glioblastoma multiforme. J Neurooncol 89:97-103

49. A randomized phase III study of temozolomide and short-course radiation versus short-course radiation alone in the treatment of newly diagnosed glioblastoma multiforme in elderly patients. Clinicaltrials.gov identifier: NCT00482677

50. Gállego Pérez-Larraya J, Ducray F, Chinot O et al (2011) Temozolomide in elderly patients with newly diagnosed glioblastoma and poor performance status: an ANOCEF phase II trial. J Clin Oncol 29(22):3050-3055

51. Wefel JS, Cloughesy T, Zazzali JL et al (2011) Neurocognitive function in patients with recurrent glioblastoma treated with bevacizumab. Neuro Oncol 13:660-668 